\title{
An Integrated Approach to Hypersonic Entry Attitude Control
}

\author{
Zhi-Qiang Pu Ru-Yi Yuan Xiang-Min Tan Jian-Qiang Yi \\ Institute of Automation, Chinese Academy of Sciences, Beijing 100190, China
}

\begin{abstract}
This paper presents an integrated approach based on dynamic inversion (DI) and active disturbance rejection control (ADRC) to the entry attitude control of a generic hypersonic vehicle (GHV). DI is firstly used to cancel the nonlinearities of the GHV entry model to construct a basic attitude controller. To enhance the control performance and system robustness to inevitable disturbances, ADRC techniques, including the arranged transient process (ATP), nonlinear feedback (NF), and most importantly the extended state observer (ESO), are integrated with the basic DI controller. As one primary task, the stability and estimation error of the second-order nonlinear ESO are analyzed from a brand new perspective: the nonlinear ESO is treated as a specific form of forced Liénard system. Abundant qualitative properties of the Liénard system are utilized to yield comprehensive theorems on nonlinear ESO solution behaviors, such as the boundedness, convergence, and existence of periodic solutions. Phase portraits of ESO estimation error dynamics are given to validate our analysis. At last, three groups of simulations, including comparative simulations with modeling errors, Monte Carlo runs with parametric uncertainties, and a six degrees-of-freedom reference entry trajectory tracking are executed, which demonstrate the superiority of the proposed integrated controller over the basic DI controller.
\end{abstract}

Keywords: Hypersonic vehicle, attitude control, dynamic inversion, active disturbance rejection control, stability analysis.

\section{Introduction}

With increasing demands for the next generation of reusable launch vehicles (RLVs), the design of practical and reliable entry guidance and control systems has received lots of attention in these years ${ }^{[1-4]}$. The goal of these systems is to plan and track an entry trajectory that stays within an entry corridor, defined by dynamic pressure, normal acceleration, heating, and controllability limits. The guidance system provides steering commands defined in terms of angle of attack and bank angle. Additionally, the sideslip angle should always be kept around zero to prevent excessive heat buildup ${ }^{[5,6]}$. The subsequent attitude system should track these three attitude commands. Entry flight has to operate over a broad flight envelope, during which the environmental and aerodynamic characteristics may change rapidly. In addition, the resulting commands from the guidance system feature rapid bank reversal maneuvers and wide ranges of angle of attack. All of these make the attitude controller design an important but challenging task.

The conventional approach to entry flight control problem is the gain scheduling $(\mathrm{GS})^{[7]}$. However, due to the large entry flight envelope and complex plant characteristics, GS is time-consuming and requires intuitive engineering skills based on experience. In contrast, dynamic inversion (DI), with its straightforwardness, has become a popular methodology for aircraft flight controller design during these decades ${ }^{[1,8-11]}$. Compared with GS, which approximately linearizes vehicle models at a set of trimmed points, DI can exactly cancel the model nonlinearities and replace undesirable dynamics with desirable dynamics using nonlin-

Manuscript received January 30, 2013; revised July 1, 2013

This work was supported by National Natural Science Foundation of China (Nos.61273149, 61203003) and the Special Project for Innovation Methods of MOST (Nos. 2012IM010200 and B1320133020). ear coordinate transformation. However, cancellation and replacement are achieved only when all exact knowledge of the model dynamics is available. In practice this assumption is not realistic because disturbances exist inevitably. Thus, extra efforts are made to deal with the influences of disturbances, resulting in diverse robust DI control methods, such as [10] with adaptive sliding mode and [11] with neural network compensation.

Active disturbance rejection control (ADRC) ${ }^{[12-15]}$ has a unique characteristic of actively rejecting both internal and external disturbances. Derived from the wellknown proportional-integral-differential (PID) control theory, ADRC fills the significant gap between control theory and practice, and shows better theoretical and practical performance than PID. The centerpiece of ADRC is taking all internal and external disturbances as a new extended state and directly estimating and compensating it using an extended state observer (ESO). By adopting nonlinear structures, ESO shows a high estimation efficiency. Besides ESO, ADRC usually includes other two components: an arranged transient process (ATP) and a nonlinear feedback (NF). Because of its simplicity and practicability, ADRC has been widely applied in recent years ${ }^{[16-18]}$.

On the other hand, there are only a few researches which combine the advantages of both DI and ADRC in diverse control applications. Such literatures include [19] on reusable launch vehicle, [20] on flying boat, and [21] on tiltrotor unmanned aerial vehicle. In this work, we integrate DI with ADRC to solve the entry attitude control problem for a much more complex plant, i.e., the generic hypersonic vehicle $(\mathrm{GHV})^{[22-24]}$. The vehicle is described by a high-fidelity model involving complex coupling effects and covering a large flight envelope. DI is used to cancel 
the nonlinearities of the known model to form a basic controller; ADRC is further integrated with DI to estimate and compensate unknown disturbances and improve the controller performance and robustness, constructing an integrated controller. Besides the vehicle model, this work features the following aspects:

1) Not only ESO but also ATP and NF are applied to both fast-state and slow-state loops to further improve the inversion controller. 2) A six degrees-of-freedom (6-DOF) reference mission for GHV is executed, involving an integrated guidance and control system design. 3) A primary contribution of this work is to investigate the stability and estimation error of the second-order nonlinear ESO with different kinds of bounded disturbances. From a brand new perspective, i.e., the nonlinear ESO is treated as a specific form of forced Liénard system ${ }^{[25-27]}$, abundant qualitative properties of the Liénard system are utilized to yield comprehensive results on nonlinear ESO solution behaviors, such as the boundedness, convergence, and existence of periodic solutions which have not yet be obtained in other researches $^{[28-30]}$.

This paper is organized as follows. The hypersonic vehicle entry model, together with the 6-DOF reference mission, is introduced in Section 2. In Section 3, DI is adopted to build a basic attitude controller. Section 4 contains the main work of this paper. With a definition of pseudo controls, the attitude system is converted into six first-order decomposed units. Then ADRC techniques including ESO, ATP, and NF are integrated with the basic DI controller. The stability analysis of the nonlinear ESO is also discussed in this section. In Section 5, three groups of simulation are executed, including comparative simulations with modeling errors, Monte Carlo runs with parametric uncertainties, and a 6-DOF reference entry trajectory tracking. At last, conclusion is given in Section 6 .

\section{Hypersonic vehicle entry problem}

\section{$2.1 \quad$ Simulation model}

GHV originally came from the idea of developing a horizontal takeoff and landing, single-stage-to-orbit (SSTO), airbreathing launch vehicle ${ }^{[22-24]}$. Its fuselage is modeled as an axisymmetric conical body. Before the entry mission, the engine is shut down. Since it is equipped with no reaction-control system, GHV will be only controlled by the aerodynamic-control surfaces: two trailing edge elevons (deflection angles $\delta_{a}$ and $\delta_{e}$, positive up) and a full span rudder (deflection angle $\delta_{r}$, positive left). Small canards are also configured for improving longitudinal stability and control performance, but they are only deployed at subsonic speeds, thus ignored in entry flight. More details about GHV can be found in [22]. The entry dynamics of this unpowered vehicle can be described as ${ }^{[31]}$

$$
\begin{gathered}
\dot{h}=V \sin \gamma \\
\dot{V}=\frac{-D+Y \sin \beta-m g \sin \gamma}{m}
\end{gathered}
$$

$$
\begin{gathered}
\dot{\gamma}=\frac{1}{m V}\left[L \cos \mu-m\left(g-\frac{V^{2}}{r}\right) \cos \gamma-Y \sin \mu \cos \beta\right] \\
\dot{\alpha}=q-\tan \beta(p \cos \alpha+r \sin \alpha)+\frac{-L+m g \cos \gamma \cos \mu}{m V \cos \beta} \\
\dot{\beta}=p \sin \alpha-r \cos \alpha+\frac{Y \cos \beta+m g \cos \gamma \sin \mu}{m V} \\
\dot{\mu}=\frac{p \cos \alpha+r \sin \alpha}{\cos \beta}+\frac{1}{m V}(L \tan \gamma \sin \mu+L \tan \beta+ \\
Y \tan \gamma \cos \mu \cos \beta-m g \cos \gamma \cos \mu \tan \beta) \\
\dot{p}=\frac{\bar{L}+\left(I_{y}-I_{z}\right) q r}{I_{x}} \\
\dot{q}=\frac{\bar{M}+\left(I_{z}-I_{x}\right) p r}{I_{y}} \\
\dot{r}=\frac{\bar{N}+\left(I_{x}-I_{y}\right) p q}{I_{z}}
\end{gathered}
$$

Here, (1)-(3) govern the translational motion where $h, V$, and $\gamma$ respectively denote the altitude, velocity, and flightpath angle. (4)-(9) govern the rotational motion. With the time-scale separation theory, the angle of attack $(\alpha)$, sideslip angle $(\beta)$, and bank angle $(\mu)$ are viewed as slow states, while the roll, pitch, and yaw rates $(p, q$, and $r$, respectively) are taken as fast states. In addition, $L, D$, and $Y$ respectively denote the lift, drag, and side forces, $\bar{L}, \bar{M}$, and $\bar{N}$ are the roll, pitch, and yaw moments, and the moments of inertia are respectively denoted by $I_{x}, I_{y}$, and $I_{z}$. As GHV is an axisymmetric plant, all products of inertia are zero.

All aerodynamic data can be found in [22] in the form of figures. Based on these figures, [23] developed their analytical expressions as up to fifth-order polynomials. These highorder expressions represent a high-fidelity model of GHV which covers the whole flight envelope and contains complex coupling effects. This high-fidelity model is adopted in this paper as the simulation plant for controller design verification. One thing should be noted that in the later fast-state loop controller design procedure, the high-order moment polynomials are approximated by first-order Taylor expansions updated at every sampling step, resulting in an affine nonlinear form to make DI applicable. The detail can be found in [32].

For the atmospheric data, the 1976 Committee on Extension to the Standard Atmosphere (COESA) model is used to compute the temperature, speed of sound, air pressure, and air density. The actuators of the control surfaces are modeled as first-order low-pass filters with certain gains. Limits on deflections and rates are separately set as

$$
\begin{gathered}
-30^{\circ} \leqslant \delta_{a}, \delta_{e}, \delta_{r} \leqslant 30^{\circ} \\
-180^{\circ} / \mathrm{s} \leqslant \dot{\delta}_{a}, \dot{\delta}_{e}, \dot{\delta}_{r} \leqslant 180^{\circ} / \mathrm{s} .
\end{gathered}
$$

\subsection{Reference mission}

The reference mission is to track a nominal entry trajectory defined in the velocity-altitude space. This was previously done in our earlier work $^{[32]}$, so we only address the main points here. Constraints on the nominal trajectory 
define an entry corridor. To emphasize the attitude control rather than the trajectory planning problem, we make two simplifications: one is to design a simple straight line from the initial point ( $h=46540 \mathrm{~m}, V=4590 \mathrm{~m} / \mathrm{s}$ ) to the terminal area energy management (TAEM) point $(h=26500 \mathrm{~m}$, $V=1250 \mathrm{~m} / \mathrm{s}$ ) as the nominal trajectory; the other is to ignore the lateral guidance issues. Based on the vehicle characteristics, a profile for the angle of attack is designed off-board before the mission as (in rad)

$$
\alpha_{c}=\left\{\begin{array}{lc}
0.25, & M a \geqslant 7 \\
0.25-0.0065(M a-7)^{2}, & 4<M a<7 .
\end{array}\right.
$$

In addition, the sideslip angle is required to be around zero. Thus only the bank angle directly results from the guidance system, where a guidance DI controller is applied. The nominal trajectory and the corresponding attitude commands are given in Fig. 1. Details can be found in $[32]$.
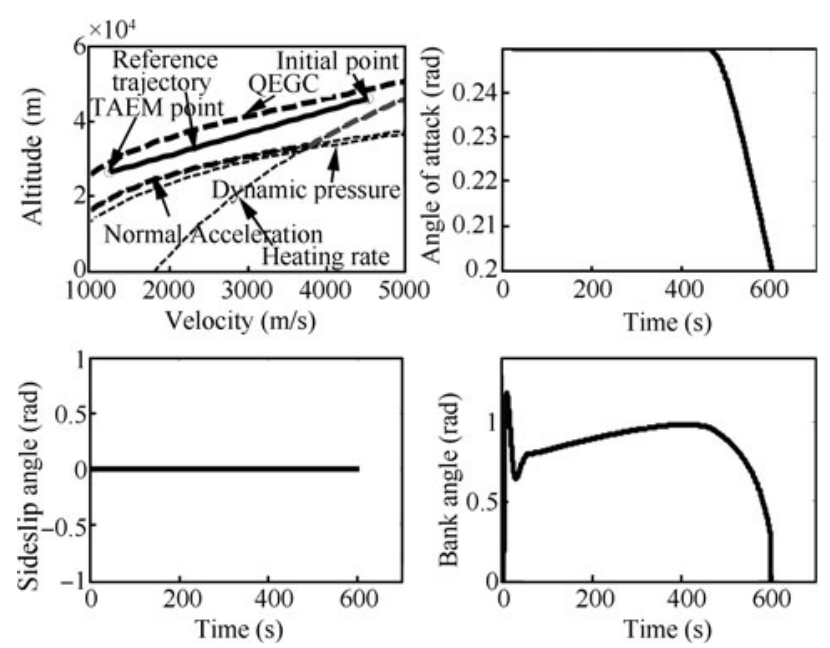

Fig. 1 Reference trajectory and the corresponding attitude commands

\section{Dynamic inversion}

In this section, DI is applied to design two basic controllers for both the fast-state and slow-state loops. A thorough theoretical discussion on DI can be found in [33]. Here it is omitted due to page limitation.

The slow-state loop (governed by (4)-(6)) can be directly written into an affine nonlinear form as

$$
\left[\begin{array}{c}
\dot{\alpha} \\
\dot{\beta} \\
\dot{\mu}
\end{array}\right]=\left[\begin{array}{c}
f_{\alpha} \\
f_{\beta} \\
f_{\mu}
\end{array}\right]+g_{s}\left[\begin{array}{c}
p \\
q \\
r
\end{array}\right]
$$

where

$$
\begin{gathered}
f_{\alpha}=\frac{-L+m g \cos \gamma \cos \mu}{m V \cos \beta} \\
f_{\beta}=\frac{Y \cos \beta+m g \cos \gamma \sin \mu}{m V}
\end{gathered}
$$

$f_{\mu}=$

$\frac{L \tan \gamma \sin \mu+L \tan \beta+Y \tan \gamma \cos \mu \cos \beta-m g \cos \gamma \cos \mu \tan \beta}{m V}$

$$
g_{s}=\left[\begin{array}{ccc}
-\cos \alpha \tan \beta & 1 & -\sin \alpha \tan \beta \\
\sin \alpha & 0 & -\cos \alpha \\
\cos \alpha \sec \beta & 0 & \sin \alpha \sec \beta
\end{array}\right] .
$$

It is obvious that the relative degree $\rho_{\alpha}=\rho_{\beta}=\rho_{\mu}=1$, and the total relative degree $\rho_{s}=3$, thus there exists no zero dynamics. Then the slow-state loop control law (denoted by $\left.\left[p_{c}, q_{c}, r_{c}\right]^{\mathrm{T}}\right)$ can be selected as

$$
\left[\begin{array}{l}
p_{c} \\
q_{c} \\
r_{c}
\end{array}\right]=g_{s}^{-1}\left(\left[\begin{array}{l}
v_{\alpha} \\
v_{\beta} \\
v_{\mu}
\end{array}\right]-\left[\begin{array}{l}
f_{\alpha} \\
f_{\beta} \\
f_{\mu}
\end{array}\right]\right)
$$

where $v_{\alpha}, v_{\beta}$, and $v_{\mu}$ are pseudo inputs that can be designed with diverse linear system design methods. Substituting (14) into (13) yields a decoupled linear system:

$$
\left[\begin{array}{lll}
\dot{\alpha} & \dot{\beta} & \dot{\mu}
\end{array}\right]^{\mathrm{T}}=\left[\begin{array}{lll}
v_{\alpha} & v_{\beta} & v_{\mu}
\end{array}\right]^{\mathrm{T}} .
$$

In this work, $P(I)$ synthesis is applied to obtain the pseudo inputs:

$$
\begin{gathered}
v_{\alpha}=\omega_{\alpha}\left(\alpha_{c}-\alpha\right)+k_{\alpha} \int\left(\alpha_{c}-\alpha\right) \mathrm{d} t \\
v_{\beta}=\omega_{\beta}\left(\beta_{c}-\beta\right) \\
v_{\mu}=\omega_{\mu}\left(\mu_{c}-\mu\right)
\end{gathered}
$$

where $\alpha_{c}, \beta_{c}$, and $\mu_{c}$ denote the reference attitude commands generated by the guidance system. $\omega_{\alpha}, \omega_{\beta}$, and $\omega_{\mu}$ are the bandwidths of the slow states, while $k_{\alpha}$ is the gain of the integral item which is particularly added to eliminate steady tracking errors. The necessity of the integral item depends on practical control performance.

The fast-state loop inversion controller is designed similarly to the slow-state one. However, since the aerodynamic moment coefficients, as mentioned in Section 2.1, are highorder polynomials instead of linear expressions of the deflection angles, (7)-(9) cannot be directly transformed into an affine nonlinear form. To solve this problem, we use firstorder Taylor expansions computed at every sampling step to approximate these coefficients. Then an affine nonlinear form of the fast-state loop similar to (13) is obtained and consequently dynamic inversion can be applied. The details are omitted here and can be found in [32].

\section{Active disturbance rejection control}

The basic DI attitude controllers show simplicity and good performance under ideal conditions. In practice, however, they may be degraded. To be more specific, three issues that may degrade these DI controllers should be addressed:

1) DI can only cancel the known nonlinearities of the model; thus when disturbances such as parametric uncertainties and modeling errors appear, the controller may fail.

2) The reference commands resulting from the guidance system may jump sharply. To track these primitive commands may cause actuator saturation when it is beyond its control ability. 
3) DI often incorporates with conventional PID to design the pseudo inputs such as (16)-(18) which are fundamentally a linear combination of the proportional, integral, and differential errors. This linear combination shows low efficiency and there may be better nonlinear forms.

In this section, we use ESO, ATP, and NF to separately solve the aforementioned three problems, resulting in a better controller integrating DI and ADRC. Attention is mainly focused on the design and stability analysis of nonlinear ESO because it is the most important component of the ADRC.

\subsection{Extended state observer design}

Disturbances are inevitable in practice. Consider the slow-state loop (13), and assume disturbances appear in all three units. If we define a "pseudo control" $U_{s}=$ $\left[\begin{array}{lll}U_{\alpha} & U_{\beta} & U_{\mu}\end{array}\right]^{\mathrm{T}}=g_{s}\left[\begin{array}{lll}p & q & r\end{array}\right]^{\mathrm{T}}$, then (13) is transformed into three decoupled single-input-single-output (SISO) systems as

$$
\left\{\begin{aligned}
\dot{\alpha} & =f_{\alpha}+U_{\alpha}+\Delta_{\alpha} \\
\dot{\beta} & =f_{\beta}+U_{\beta}+\Delta_{\beta} \\
\dot{\mu} & =f_{\mu}+U_{\mu}+\Delta_{\mu}
\end{aligned}\right.
$$

where $\Delta_{\alpha}, \Delta_{\beta}$, and $\Delta_{\mu}$ respectively denote the total disturbance in each channel, including parametric uncertainties and modeling errors.

The core idea of ESO is to take total disturbance as a new system state, and then establish a state observer to estimate and compensate this disturbance. Consider the first equation of (19). Treat $\Delta_{\alpha}$ as an extended state and suppose we have $\dot{\Delta}_{\alpha}=-w_{\alpha}(t)$ with $w_{\alpha}(t)$ unknown but bounded. Define new states as $x_{\alpha 1}=\alpha$ and $x_{\alpha 2}=\Delta_{\alpha}$. Then the angle of attack dynamics can be written as a second-order extended system:

$$
\left\{\begin{array}{l}
\dot{x}_{\alpha 1}=x_{\alpha 2}+f_{\alpha}+U_{\alpha} \\
\dot{x}_{\alpha 2}=-w_{\alpha}(t) \\
y=x_{\alpha 1}
\end{array}\right.
$$

An ESO can be established for (20) as follows:

$$
\left\{\begin{array}{l}
e=z_{\alpha 1}-y \\
\dot{z}_{\alpha 1}=z_{\alpha 2}+f_{\alpha}+U_{\alpha}-\beta_{\alpha 1} f_{1}(e) \\
\dot{z}_{\alpha 2}=-\beta_{\alpha 2} f_{2}(e)
\end{array}\right.
$$

where $z_{\alpha i}$ is the estimation value of $x_{\alpha i}, \beta_{\alpha i}>0$ is the observer gain, and $f_{i}(e)$ is an appropriate function of estimation error (here $i=1,2)$. Particularly, we select $f_{1}(e)=e$ and $f_{2}(e)=f a l\left(e, \alpha_{\alpha}, \delta_{\alpha}\right)$ where $f a l$ is in a nonlinear form $^{[14]}$ :

$$
f a l\left(e, \alpha_{\alpha}, \delta_{\alpha}\right)= \begin{cases}|e|^{\alpha_{\alpha}} \operatorname{sgn}(e), & |e|>\delta_{\alpha} \\ \frac{e}{\delta_{\alpha}^{1-\alpha_{\alpha}}}, & |e| \leqslant \delta_{\alpha}\end{cases}
$$

where $\delta_{\alpha} \geqslant 0$ and often $\alpha_{\alpha}>0$. In (22), the nonlinear structure $\left(0<\alpha_{\alpha}<1\right)$ shows a much higher estimation efficiency than the linear form $\left(\alpha_{\alpha}=1\right)$. According to [14], if the parameters $\beta_{\alpha 1}, \beta_{\alpha 2}, \alpha_{\alpha}$, and $\delta_{\alpha}$ are properly chosen, the observer (21) can estimate the real-time values of the original states with a satisfying accuracy, that is, $z_{\alpha 1} \rightarrow \alpha, z_{\alpha 2} \rightarrow \Delta_{\alpha}$.
Such ESOs can also be designed for the sideslip angle and bank angle channels to yield $z_{\beta 1} \rightarrow \beta, \quad z_{\beta 2} \rightarrow \Delta_{\beta}$ and $z_{\mu 1} \rightarrow \mu, z_{\mu 2} \rightarrow \Delta_{\mu}$. The estimation values $z_{s}=$ $\left[\begin{array}{lll}z_{\alpha 2} & z_{\beta 2} & z_{\mu 2}\end{array}\right]^{\mathrm{T}}$ can then be applied to compensate the disturbances in (19). If we choose the pseudo control as

$$
U_{s}=\left[\begin{array}{l}
v_{\alpha} \\
v_{\beta} \\
v_{\mu}
\end{array}\right]-\left[\begin{array}{l}
f_{\alpha} \\
f_{\beta} \\
f_{\mu}
\end{array}\right]-z_{s}
$$

then (19) can be transformed into a similar form to (15), which means the effect of disturbances is completely compensated. Subsequently, the actual control law for the slowstate loop is obtained as

$$
\left[\begin{array}{lll}
p_{c} & q_{c} & r_{c}
\end{array}\right]^{\mathrm{T}}=g_{s}^{-1} U_{s} .
$$

We must stress that in (19) the known dynamics $f_{\alpha}, f_{\beta}$, and $f_{\mu}$ can also be included into the disturbance items to be estimated for the sake of simplicity, and ESO can still estimate them. However, in order to reduce the burden of $\mathrm{ESO}$, we retain the function form of the known part to obtain "nominal values" such that ESO only needs to estimate the disturbance part.

Finally, the fast-state loop also needs three ESOs to estimate and compensate the disturbances. The design process is omitted because it is similar to that of the slow-state loop.

\subsection{Arranged transient process and non- linear feedback}

The reference attitude commands resulting from the guidance system often contains huge jumps. To track such primitive commands may be beyond the control ability of the attitude controller and result in actuator saturation. In practice, it is necessary to arrange a proper transient process that the output of the plant can reasonably follow. Such an operation can solve the conflicts between rapidity and overshoot. ADRC offers several ATP techniques, such as a function generator and an extended state observer.

In this work, ATP is constructed in a discrete-time form:

$$
\left\{\begin{aligned}
\eta_{1}(k+1)= & \eta_{1}(k)+\tau \eta_{2}(k) \\
\eta_{2}(k+1)= & \eta_{2}(k)+\tau \operatorname{fhan}\left(\eta_{1}(k)-\right. \\
& \left.\eta(k), \eta_{2}(k), r_{0}, \tau_{0}\right)
\end{aligned}\right.
$$

where $\tau$ stands for the sampling period, $\eta$ is the state of the original process, while $\eta_{1}$ and $\eta_{2}$ are the states of the arranged process and its derivative with initial values $\eta_{1}(0)=\eta(0)$ and $\eta_{2}(0)=0$. Here, the function fhan is the time-optimal control law for a discrete double integral plant and its expression can be found in [14]. The control parameters $r_{0}$ and $\tau_{0}$ are separately called "speed factor" and "filter factor". Increasing or decreasing $r_{0}$ can speed up or slow down the transient process, thus it is selected according to the plant characteristics. Changing $\tau_{0}$ can affect the filtering effect on the original process. In most cases, $\tau_{0}$ is set equal to the sampling period $\tau$.

Another problem of the basic DI controllers is that the pseudo inputs in (16)-(18) are synthesized using conventional PID, which employs a linear combination of the error, as well as its integration and differentiation. In fact, 
NF is a better choice in improving control performance and rejecting disturbances ${ }^{[12,14]}$. Using the nonlinear function fal $(e, \alpha, \delta)$ defined in (22), we replace (16)-(18) by

$$
\begin{aligned}
v_{i}= & k_{0 i} f a l\left(e_{0 i}, \alpha_{0 i}, \delta_{i}\right)+k_{1 i} f a l\left(e_{1 i}, \alpha_{1 i}, \delta_{i}\right)+ \\
& k_{2 i} f a l\left(e_{2 i}, \alpha_{2 i}, \delta_{i}\right)
\end{aligned}
$$

where $i=\alpha, \beta$ or $\mu$. $e_{0 i}$ denotes the tracking error, while $e_{1 i}$ and $e_{2 i}$ denote its integration and differentiation, respectively. The control parameters $k_{0 i} \sim k_{2 i}, \alpha_{0 i} \sim \alpha_{2 i}$, and $\delta_{i}$ offer a flexible way to simultaneously meet multiple control requirements. Take $\alpha_{0 i} \sim \alpha_{2 i}$ for example. A general rule lies in that: choosing $0<\alpha_{0 i}<1$ may decrease the steady tracking error; choosing $0<\alpha_{1 i}<1$ may avoid integral windup; choosing $\alpha_{2 i}>1$ may restrain the overshoot. In practice, the necessity of the integral or differentiation item depends on actual control performance. The graphical interpretation of $\operatorname{fal}(e, \alpha, \delta)$ with different parameters $\alpha$ is illustrated in Fig. 2.

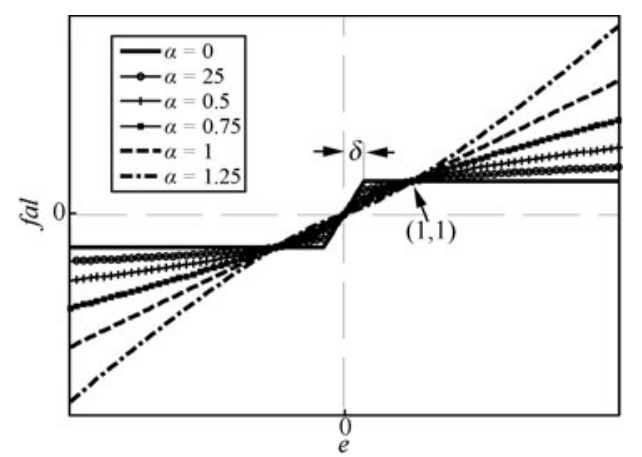

Fig. 2 Nonlinear function $\operatorname{fal}(e, \alpha, \delta)$ with different values of parameter $\alpha$

So far, we have constructed an attitude controller that integrates DI with three ADRC components: ESO, ATP, and NF. The whole attitude control scheme is shown in Fig. 3. The most important components ESO1 and ESO2 separately contain three extended state observers. Because the inner states vary fast, ATP2 is optional. The guidance system can be found in [32] and is omitted here, but should be added outside the attitude system in the reference mission simulation. For each attitude loop, the overall design procedure and key points can be concluded as follows (here $i=\alpha, \beta, \mu, p, q, r)$ :
Step 1. Transform the vehicle dynamics into a nonlinear affine form and derive a basic DI control law with linear PID combination.

Step 2. Design a second-order ESO for each channel for uncertainty estimation. The key work is to tune the parameters $\beta_{i 1}, \beta_{i 2}$, and $\alpha_{i}$.

Step 3. Arrange a proper transient process for each primitive attitude command according to the vehicle characteristics. The key work is to determine the speed factor $r_{0}$ and the filter factor $\tau_{0}$.

Step 4. Replace the linear PID combination in the basic DI control law by NF. The proportional item is usually necessary, while the integral and differential items may be optional. The control parameters to be tuned include $k_{0 i} \sim k_{2 i}, \alpha_{0 i} \sim \alpha_{2 i}$, and $\delta_{i}$.

Step 5. Conduct robust simulation tests and analyze the results. Accordingly, make some proper improvements of the overall scheme.

Remark 1. Parameters for ESO, ATP, and NF are relatively independent of each other, which makes the parameter tuning much easier. For example, if the parameters in ESO are tuned such that the uncertainties are well observed, they will not be changed again during the tuning of other parameters. In addition, as reported in [14], ADRC exhibits great robustness to control parameter variations, indicating its good parameter adaption property.

\subsection{ESO stability and estimation error analysis}

The primary advantage of the integrated controller over the basic DI controller lies in its sound property of disturbance rejection, which is chiefly due to the application of ESO. The final problem is, how accurate can ESO estimate the disturbance? In this section, we will analyze the stability and estimation error of the nonlinear ESO from a brand new perspective.

For linear ESOs, many useful results on stability analysis have been obtained ${ }^{[34,35]}$. For nonlinear ESOs, however, conventional theories are difficult to be applied. In the literature, two dominant approaches are adopted, i.e., the self stable region (SSR) theory ${ }^{[28]}$ and the Lyapunov method $^{[29,30]}$, resulting in some sufficient conditions on the stability behavior for a specified kind of disturbance. However, the tedious derivation and strict constraints make these conclusions difficult to be expanded. In fact, they are

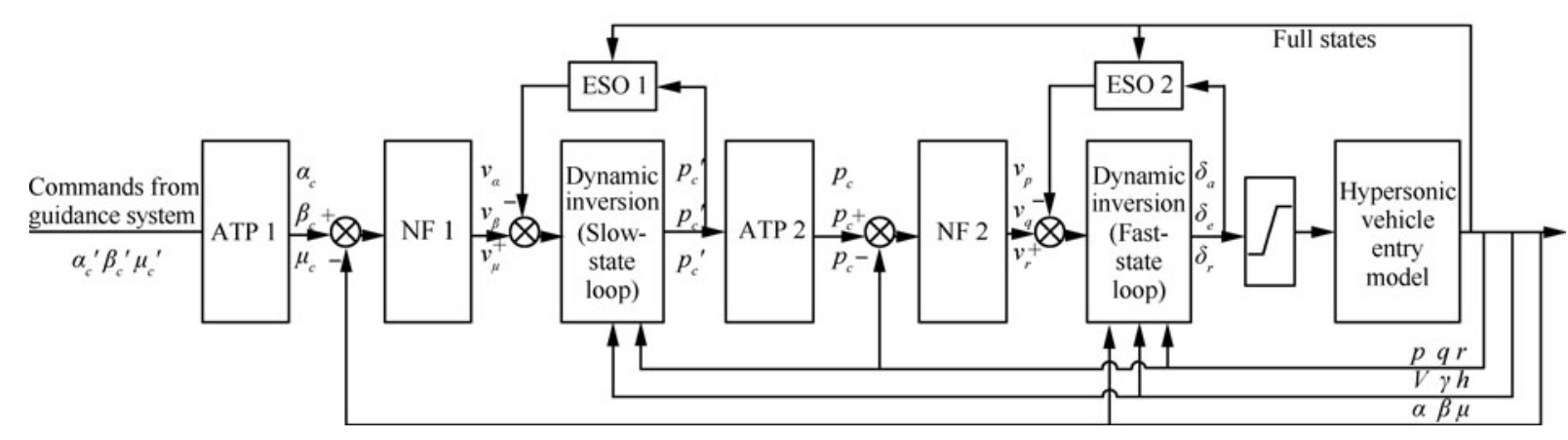

Fig. 3 The whole configuration of the integrated attitude control scheme 
even not practical because these sufficient conditions may enlarge the estimation errors for many practical cases. In this work, we take a brand new but easier way to investigate the second-order nonlinear ESO by treating it as a generalized forced Liénard system. For this well-known system, mathematicians and physicists have obtained many interesting results on its boundedness, stability, and existence of periodic solutions and almost periodic solutions. The interested reader is advised to refer to [25-27] and the references quoted therein. Next, we utilize these qualitative properties to obtain some more comprehensive results on nonlinear ESO stability and estimation error than those in [28-30], particularly on the asymptotic behavior of solutions with disturbances that are absolutely integrable.

Let us start with the ESO of the angle of attack unit. The extended system and established ESO are respectively expressed by (20) and (21). As already discussed, if we select a proper group of parameters $\beta_{\alpha 1}, \beta_{\alpha 2}, \alpha_{\alpha}$, and $\delta_{\alpha}$, we have $z_{\alpha 1} \rightarrow x_{\alpha 1}, z_{\alpha 2} \rightarrow x_{\alpha 2}$. Here we define estimation errors $e_{1}=z_{\alpha 1}-x_{\alpha 1}$ and $e_{2}=z_{\alpha 2}-x_{\alpha 2}$. According to (20) and (21), the error dynamics are obtained as

$$
\left\{\begin{array}{l}
\dot{e}_{1}=e_{2}-\beta_{\alpha 1} e_{1} \\
\dot{e}_{2}=w_{\alpha}(t)-\beta_{\alpha 2} \operatorname{fal}\left(e_{1}, \alpha_{\alpha}, \delta_{\alpha}\right) .
\end{array}\right.
$$

Particularly, the disturbance $w_{\alpha}(t)$ is supposed to be continuous and absolutely integrable. System (27) is a specific form of generalized Liénard system with forcing item $w_{\alpha}(t)$. Define a linear transformation as

$$
\left\{\begin{array}{l}
z_{1}=e_{1} \\
z_{2}=e_{2}-\beta_{\alpha 1} e_{1}
\end{array}\right.
$$

Then (27) is transformed into an equivalent system

$$
\left\{\begin{array}{l}
\dot{z}_{1}=z_{2} \\
\dot{z}_{2}=-\beta_{\alpha 2} \operatorname{fal}\left(z_{1}, \alpha_{\alpha}, \delta_{\alpha}\right)-\beta_{\alpha 1} z_{2}+w_{\alpha}(t) .
\end{array}\right.
$$

This is another specific form of Liénard systems. For this system, we have the following lemma.

Lemma 1. For system (29), choose $\beta_{\alpha 1}>0, \beta_{\alpha 2}>0$ and define $f a l$ as (22). If $w_{\alpha}(t)$ is continuous and absolutely integrable, then all solutions of (29) are uniformly bounded.

Lemma 1 is a direct derivation of the well-known conclusion obtained in [36] (Theorem 8.8 and Example 8.3), thus the proof is omitted here because our focus is mainly on the asymptotic behavior of the solutions.

Without loss of generality, consider a general form of system (29) as

$$
\left\{\begin{array}{l}
\dot{z}_{1}=z_{2} \\
\dot{z}_{2}=-f\left(z_{1}\right) z_{2}-g\left(z_{1}\right)+e(t)
\end{array}\right.
$$

where $f(\cdot)$ and $g(\cdot)$ are nonlinear functions, and $e(t)$ is the forced item. For this system, we have the following asymptotic stability theorem.

Theorem 1. For system (30), suppose the following assumptions are valid:

1) $f\left(z_{1}\right), g\left(z_{1}\right)$, and $e(t)$ are continuous;

2) $z_{1} g\left(z_{1}\right)>0\left(z_{1} \neq 0\right), g(0)=0$;
3) $f\left(z_{1}\right)>0$ and $F\left(z_{1}\right)=\int_{0}^{z_{1}} f(u) \mathrm{d} u \rightarrow \pm \infty$ as $z_{1} \rightarrow \pm \infty$

4) $E(t)=\int_{0}^{t}|e(s)| \mathrm{d} s<\infty$

Then all solutions of (30) satisfy $z_{1} \rightarrow 0, z_{2} \rightarrow 0$ as $t \rightarrow \infty$.

To prove this theorem, we consider a system

$$
\dot{x}=F(t, x)+G(t, x), \quad x \in \mathbf{R}^{n}
$$

where $F$ and $G$ are continuous vector functions on $I(0 \leqslant$ $t<\infty) \times Q\left(Q\right.$ is an open set in $\left.\mathbf{R}^{n}\right)$. The following lemma in [36] (Theorem 10.3) is needed.

Lemma 2. Suppose that there exists a non-negative continuously differentiable scalar function $V(t, x)$ on $I(0 \leqslant$ $t<\infty) \times Q$ such that $\dot{V}(t, x) \leqslant-W(x)$, where $W(x)$ is a positive definite function with respect to a closet set $\Omega$ in the space $Q$. Then all solutions of (31) approach $\Omega$. Moreover, suppose that $F(t, x)$ of system (31) is bounded for all $t$ when $x$ belongs to an arbitrary compact set in $Q$ and that $F$ satisfies the following two conditions with respect to $\Omega$ :

1) $F(t, x)$ tends to a function $H(x)$ for $x \in \Omega$ as $t \rightarrow \infty$, and on any compact set in $\Omega$ this convergence is uniform. Consequently, $H(x)$ is a continuous function on $\Omega$.

2) Corresponding to each $\varepsilon>0$ and any $y \in \Omega$, there exist a $\delta, \delta=\delta(y)>0$ and a $T, T=T(y)>0$ such that if $t \geqslant T$ and $\|x-y\|<\delta(y)$, we have that $\|F(t, x)-F(t, y)\|<\varepsilon$. Then every bounded solution of (31) approaches the largest semi-invariant set of the system $\dot{x}=H(x)$ contained in $\Omega$ as $t \rightarrow \infty$.

Now, we use Lemma 2 to prove Theorem 1.

Proof. First, as discussed for (29) in Lemma 1, the solutions of (30) are uniformly bounded according to [36] (Theorem 8.8 and Example 8.3).

Construct a continuous function for (30) as

$$
V\left(t ; z_{1}, z_{2}\right)=\mathrm{e}^{-2 E(t)}\left[G\left(z_{1}\right)+\frac{z_{2}^{2}}{2}+1\right]
$$

where

$$
G\left(z_{1}\right)=\int_{0}^{z_{1}} g(u) \mathrm{d} u \geqslant 0
$$

We have

$$
V\left(t ; z_{1}, z_{2}\right) \geqslant \mathrm{e}^{-2 E(\infty)} \frac{z_{2}^{2}}{2} \geqslant 0
$$

and

$$
\begin{aligned}
\dot{V}= & \mathrm{e}^{-2 E(t)}\left\{-2|e(t)|\left(G\left(z_{1}\right)+\frac{z_{2}^{2}}{2} 1\right)+g\left(z_{1}\right) z_{2}-\right. \\
& \left.f\left(z_{1}\right) z_{2}^{2}-g\left(z_{1}\right) z_{2}+z_{2} e(t)\right\} \leqslant \\
& \mathrm{e}^{-2 E(t)}\left\{-|e(t)|\left(z_{2}^{2}+2-\left|z_{2}\right|\right)-f\left(z_{1}\right) z_{2}^{2}\right\} \leqslant \\
& -f\left(z_{1}\right) z_{2}^{2} \mathrm{e}^{-2 E(t)} \leqslant-f\left(z_{1}\right) z_{2}^{2} \mathrm{e}^{-2 E(\infty)} .
\end{aligned}
$$

Define

$$
W\left(z_{1}, z_{2}\right)=f\left(z_{1}\right) z_{2}^{2} \mathrm{e}^{-2 E(\infty)}
$$

Then $W\left(z_{1}, z_{2}\right)$ is a positive definite function with respect to the set $\Omega_{z}=\left\{\left(z_{1}, z_{2}\right) \mid z_{2}=0\right\}$. According to Lemma 2, all solutions of (30) approach $\Omega_{z}$. Moreover, in view of (30) and (31), it follows that

$$
F(t, z)=\left[\begin{array}{c}
z_{2} \\
-f\left(z_{1}\right) z_{2}-g\left(z_{1}\right)
\end{array}\right], G(t, z)=\left[\begin{array}{c}
0 \\
e(t)
\end{array}\right]
$$


It is easy to verify the conditions 1 ) and 2) in Lemma 2. For $\left(z_{1}, z_{2}\right) \in \Omega_{z}$, as $t \rightarrow \infty$, we have

$$
F(t, z) \rightarrow H(t, z)=\left[\begin{array}{c}
0 \\
-g\left(z_{1}\right)
\end{array}\right]
$$

According to Lemma 2, every solution of (30) approaches the largest semi-invariant set of the system

$$
\left\{\begin{array}{l}
\dot{z}_{1}=0 \\
\dot{z}_{2}=-g\left(z_{1}\right)
\end{array}\right.
$$

By the condition on $g\left(z_{1}\right)$, the largest semi-invariant set contained in $\Omega_{z}$ is only the origin. Therefore, we conclude that $z_{1} \rightarrow 0$ and $z_{2} \rightarrow 0$ as $t \rightarrow \infty$.

Theorem 1 offers a general result for any Liénard system as (30) that satisfies the assumptions 1)-4) in Theorem 1. For the specific angle of attack ESO error dynamics (29), by comparing it with (30), we have

$$
\left\{\begin{array}{l}
f\left(z_{1}\right)=\beta_{\alpha 1} \\
g\left(z_{1}\right)=\beta_{\alpha 2} f a l\left(z_{1}, \alpha_{\alpha}, \delta_{\alpha}\right) \\
e(t)=w_{\alpha}(t) .
\end{array}\right.
$$

Obviously, in (29) assumptions 1)-3) do hold. So if the disturbance satisfies the assumption 4), that is, the disturbance is absolutely integrable, then the estimation errors will finally converge to zero. This is a new meaningful conclusion which is not included in other ESO stability research papers such as [28-30].

More importantly, the approach that takes the nonlinear ESO as a specific Liénard system offers a huge opportunity to introduce the abundant properties of Liénard system to nonlinear ESO, such as the boundedness, stability, and existence of periodic solutions and almost periodic solutions. For example, [37] showed that if the disturbance is a continuous periodic function, then there exists at least one periodic solution of (30). Moreover, if the system is stable, this periodic solution is unique and all other solutions approach it. [38] showed that all solutions of (30) are bounded whether $e(t)$ is absolutely integrable or bounded, where the bounded case of $e(t)$ is new. In addition, [27] also obtained a similar convergence property with a weaker condition on the disturbance. All these conclusions can be drawn for the nonlinear second-order ESO and a comprehensive theoretic frame can be built up for ESO stability and estimation error analysis.

One last thing that should be noted is the usefulness of the nonlinear function fal. As Theorem 1 shows, all solutions of (30) finally converge to the origin. Thus, for the steady state we have $z_{1}=z_{2}=0$ and $\dot{z}_{1}=\dot{z}_{2}=0$. In view of the transformation (28) and the original error dynamics (27), the original steady estimation error can be obtained as

$$
\left\{\begin{array}{l}
e_{1 s}=f a l^{-1}\left(\frac{\omega_{\alpha}}{\beta_{\alpha 2}}\right) \\
e_{2 s}=\beta_{\alpha 1} e_{1 s}
\end{array}\right.
$$

When $\left|w_{\alpha} / \beta_{\alpha 2}\right|<1$ (satisfied by selecting proper $\beta_{\alpha 2}$ ), it is easy to verify that the nonlinear structure $\left(\alpha_{\alpha}<1\right)$ results in a much smaller steady error bound than the linear structure $\left(\alpha_{\alpha}=1\right)$. In fact, the nonlinear function fal together with the observer gains $\beta_{\alpha 1}$ and $\beta_{\alpha 2}$ can also improve the dynamic process of the estimation error system.

Finally, three examples are executed with different kinds of disturbances:

Case 1. $w_{\alpha}(t)=0$

Case 2. $w_{\alpha}(t)=w_{0} \mathrm{e}^{-t}$

Case 3. $w_{\alpha}(t)=w_{0} \sin (t)$.

In demonstration we choose $\alpha_{\alpha}=0.5, \delta_{\alpha}=0$ for the nonlinear function fal.

In Case 1, no disturbance is added so the assumption 4) of Theorem 1 definitely holds. The phase portraits with the same initial values but different observer gains are shown in Fig. 4. Although all trajectories converge to $(0,0)$, the dynamic processes are quite different because the gains $\beta_{\alpha 1}$ and $\beta_{\alpha 2}$ have a great effect on them. Making a tradeoff between the rapidity and overshoot, we choose $\beta_{\alpha 1}=\beta_{\alpha 2}=15$ in next simulation examples and in the final attitude controllers. In Case 2, assumption 4) also holds. The solution behaviors are illustrated in Figs. 5 (a) and (b), where $w_{0}=-1.5$ in Fig. 5 (a) and $w_{0}=1.5$ in Fig. 5 (b). As is proved in Theorem 1, all solutions converge to $(0,0)$. For a periodic disturbance that does not match assumption 4 ), there exists at least one periodic solution as demonstrated by [37]. Furthermore, if the system is stable, this periodic solution is unique and all other solutions converge to it. Case 3 shows such an example. Figs. 5 (c) and (d) depict the phase portraits with $w_{0}=1.5$, where Fig. 5 (c) shows that all solutions converge to a periodic solution, and Fig. 5 (d) draws this unique periodic solution.

Remark 2. The above stability analysis is mostly based on the variables $z_{1}$ and $z_{2}$. By applying an inverse transformation of (28), we can obtain the original estimation errors $e_{1}$ and $e_{2}$.

Remark 3. As shown in (33), for a large class of disturbances, increasing $\beta_{\alpha 1}$ and $\beta_{\alpha 2}$ can notably decrease the estimation errors. However, the dynamic process may become worse when the gains go too large. Tradeoff should be made between the dynamic and steady responses in choosing $\beta_{\alpha 1}$ and $\beta_{\alpha 2}$.
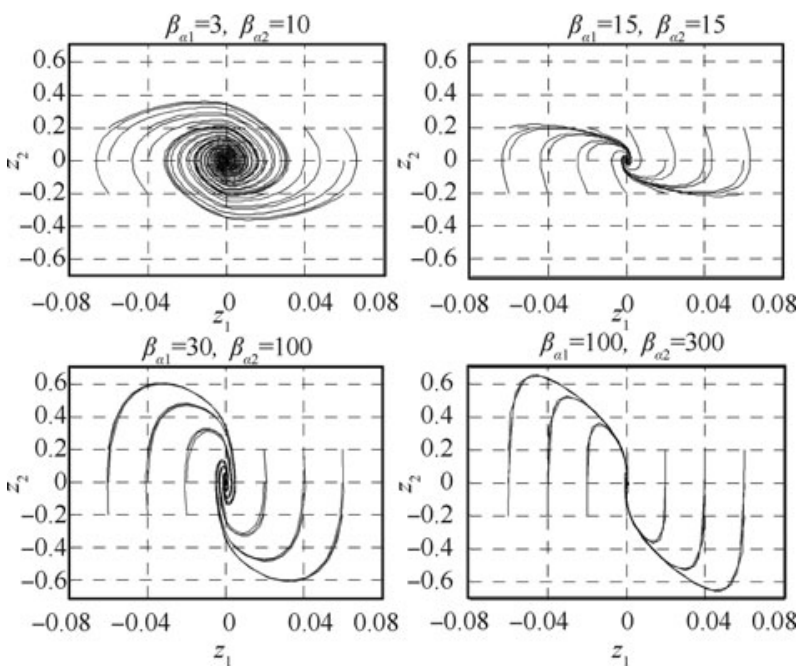

Fig. 4 Phase portraits of Case 1 with different gains 
Remark 4. By treating the nonlinear ESO as a specific Liénard system, a huge opportunity is offered to investigate the properties of ESO. The contribution in this work is not just some specific results, but more importantly a brand new perspective to investigate nonlinear ESO.

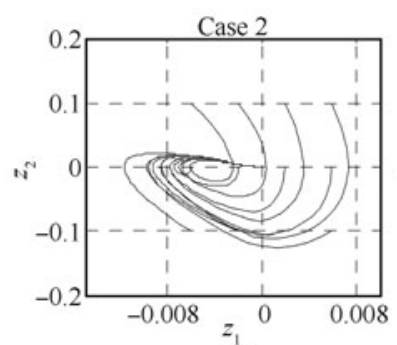

(a)

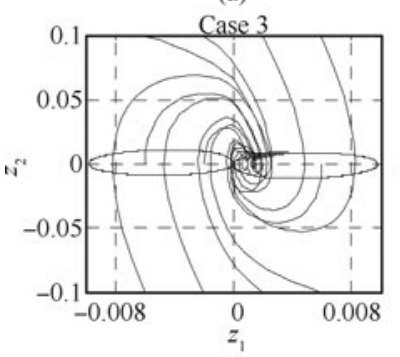

(c)

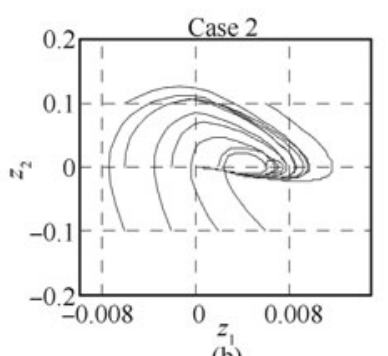

(b)

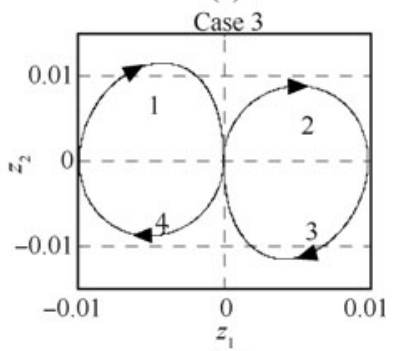

(d)
Fig. 5 Phase portraits of Case 2 and Case 3

\section{Simulation results}

Three groups of simulation are executed from three different perspectives. First, several comparative simulations between the basic DI controller and the integrated controller are carried out. Next, Monte Carlo runs are executed to demonstrate the controller robustness to parameter uncertainties. At last, the control scheme is verified in the 6DOF reference mission to indicate its applicability in an integrated guidance and control system.

\subsection{Comparative simulations}

First, comparative simulations are executed to demonstrate the advantages of the integrated controller over the basic DI controller, particularly when modeling errors are involved. The commanded attitude angles (in rad) are

$$
\left\{\begin{array}{l}
\alpha_{c}=0.2 \\
\beta_{c}=0 \\
\mu_{c}=\operatorname{sgn}(\sin (0.05 \pi t)) .
\end{array}\right.
$$

Here, $\alpha_{c}=0.2 \mathrm{rad}$ is nearly the largest angle of attack in the GHV aerodynamic database, while the square wave bank angle command simulates the bank reversal maneu- ver. Control parameters of the integrated controller mostly include those for the ESO (shown in (21) and (22)), ATP (shown in (25)), and NF (shown in (26)). Table 1 lists all these parameters for the slow states $(\alpha, \beta$, and $\mu)$ and fast states $(p, q$, and $r)$ (with sampling period $0.01 \mathrm{~s}$ ). Theoretically, each channel needs 15 parameters. However, some are optional (labeled by "/"). In addition, most parameters are the same for each channel, showing the great parameter adaption and simplicity of tuning in ADRC. In the basic DI controller, the control parameters are only $k_{0 i} \sim k_{2 i}$.

The effect of ATP is firstly verified. Take the slow-state loop for demonstration (see Fig. 6). With ATP, Fig. 6 (a) shows the original commands $\alpha_{c}^{\prime}, \beta_{c}^{\prime}, \mu_{c}^{\prime}$, the arranged processes $\alpha_{c}, \beta_{c}, \mu_{c}$, and the actual responses of the attitude angles. All attitude angles track their arranged processes well. The sideslip angle is kept around zero. During every bank reversal a small oscillation occurs in the sideslip angle, indicating strong couplings between rolling and yawing motions. Moreover, we can make the responses even faster by properly increasing the ATP speed factor. In contrast, Fig. 6 (b) shows the responses for the basic DI controller without ATP. The improper original commands cause actuator saturation and chattering, thus the controller loses its ability and the simulation is forced to be stopped within $30 \mathrm{~s}$. Therefore, ATP is an essential component, especially for the slow-state loop.

Next, the modeling error disturbance is considered. For demonstration, assume that a sine disturbance $\Delta_{\alpha}=0.03 \sin (0.2 t)$ and a square disturbance $\Delta_{\mu}=$ $0.05 \operatorname{sgn}(\sin (0.2 t))$ separately describe the total modeling errors existing in the angle of attack and the bank angle channels. The comparison simulation is depicted in Figs. 7 (a) and (b). Here, the ATP curves are canceled for brevity. It is seen that although the basic DI controller does not completely lose its control ability, the control performance is severely degraded, where an oscillation appears in the angle of attack response and a tracking error up to 2 degree appears in the bank angle response. On the contrary, the integrated controller exhibits a satisfying disturbance rejection ability due to ESO. Figs. 7 (c) and (d) show the actual and observed disturbance values in these two channels, respectively. ESO shows a good estimation ability. If we keep on increasing the amplitude or frequency of the disturbances, the basic DI controller completely loses its control ability while the integrated controller still performs well. Such disturbances can be added into the fast-state dynamics and in these situations the integrated controller also shows great advantages over the basic DI controller.

At last, two comparative tests for NF and linear feedback (LF) are conducted. In the first case, no disturbance is added. Parameters for NF are still set as listed in Table 1.

Table 1 Control parameters in the integrated controller

\begin{tabular}{|c|c|c|c|c|c|c|c|c|c|c|c|}
\hline \multirow{2}{*}{ Channel } & \multicolumn{2}{|c|}{ ATP } & & \multicolumn{4}{|c|}{$\mathrm{NF}$} & \multicolumn{4}{|c|}{$\mathrm{ESO}$} \\
\hline & $r_{0}$ & $\tau_{0}$ & $k_{0 i}$ & $\begin{array}{r}\mathrm{P} \\
\alpha_{0 i} \\
\end{array}$ & $\delta_{0 i}$ & $\begin{array}{c}\mathrm{I} \\
k_{1 i}, \alpha_{1 i}, \delta_{1 i}\end{array}$ & $\begin{array}{c}\mathrm{D} \\
k_{2 i}, \alpha_{2 i}, \delta_{2 i}\end{array}$ & $\beta_{i 1}$ & $\beta_{i 2}$ & $\alpha_{i}$ & $\delta_{i}$ \\
\hline Slow states & 0.1 & 0.05 & 1 & 0.85 & 0.05 & 1 & / & 15 & 15 & 0.5 & 0.01 \\
\hline Fast states & / & / & 4 & 0.85 & 0.05 & / & / & 15 & 15 & 0.5 & 0.01 \\
\hline
\end{tabular}


For LF, we use the same gains $k_{0 i}$ while set $\alpha_{0 i}=1$ (indicating an LF structure). Fig. 8 (a) shows the comparative responses of angle of attack. It is seen that with the same control gains NF shows a faster response than the LF. In the second case, assume that a disturbance $\Delta_{\alpha}=0.03 \sin (0.2 t)$ exists in the angle of attack channel. Suppose uncertainty compensation of ESO is canceled. Responses for NF (two runs, setting $\alpha_{0 i}=0.85$ and $\alpha_{0 i}=0.65$, respectively) and LF are given in Fig. 8 (b). Obviously, a smaller $\alpha_{0 i}$ (indicating a larger nonlinearity structure) provides a better disturbance rejection ability. However, additionally considering dynamic response features, we choose $\alpha_{0 i}=0.85$ for the final controller. These two cases show that, as already discussed for (26), proper NF can feasibly improve control performance over LF. The basic reason lies in that NF provides a nonlinear mechanism that completely agrees with the intuition obtained from practical engineering experiences. For example, when $0<\alpha_{0 i}<1$, it provides higher control gains when error is small and lower control gains when error is large. This shows the similar idea to many fuzzy logic or gain scheduling methods, while remains in a much simpler form.
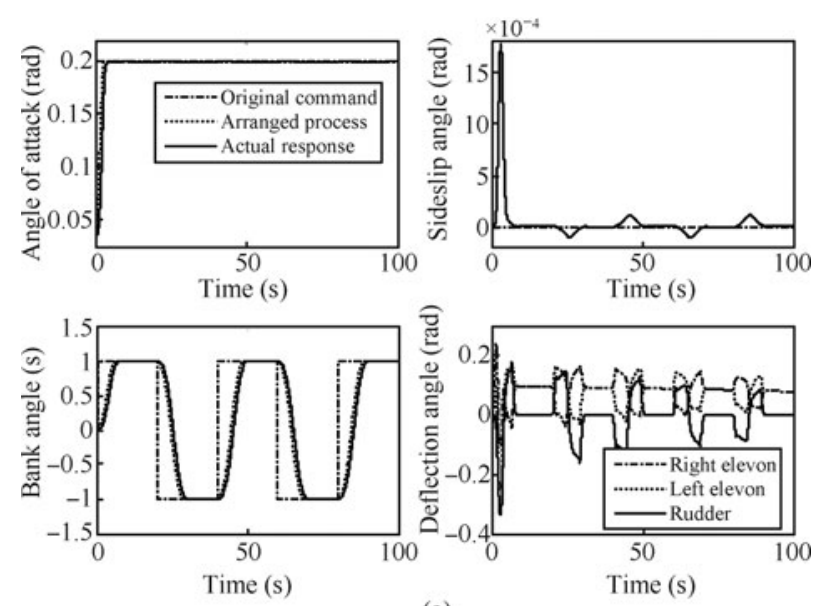

(a)
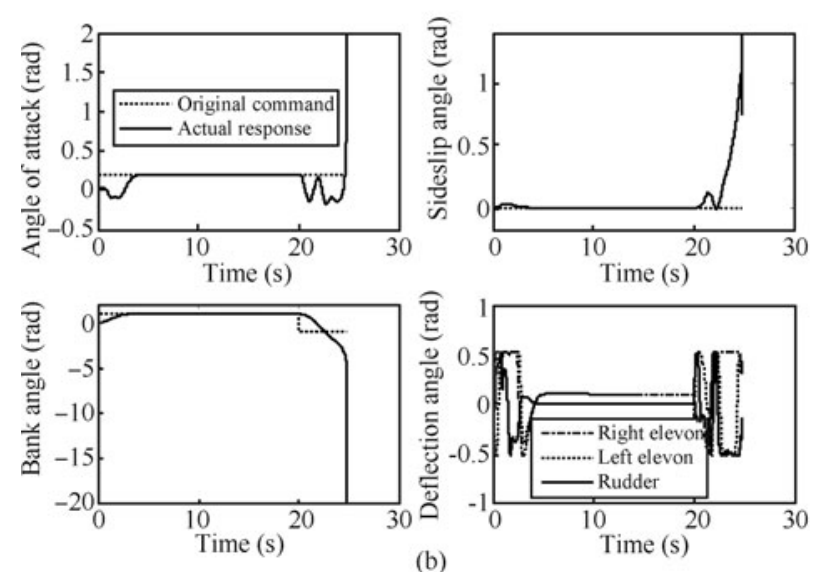

Fig. 6 Comparative simulation for ATP effect verification. (a) with ATP; (b) without ATP

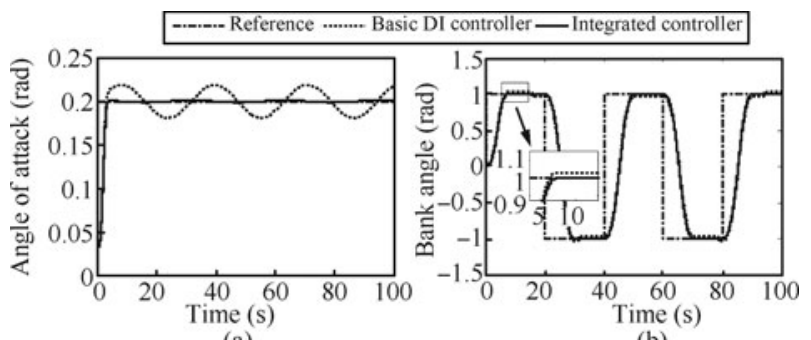

(a)

(b)

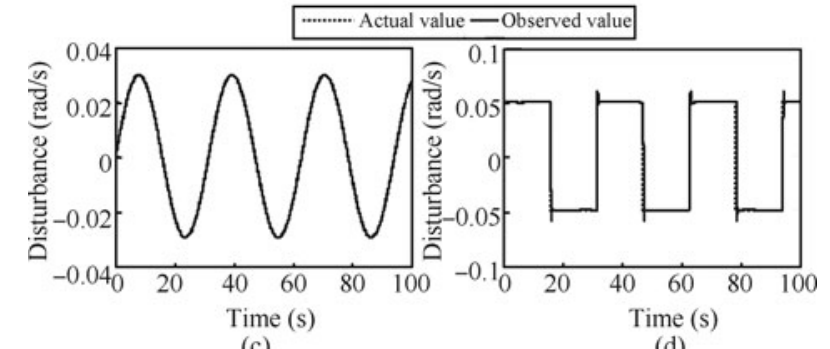

(c)

(d)

Fig. 7 Responses with modeling errors existing in the angle of attack and bank angle dynamics

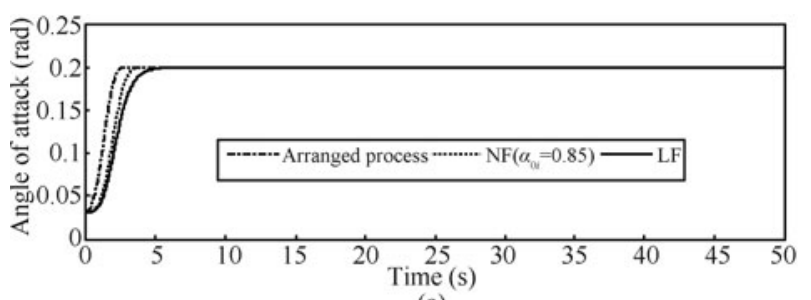

(a)

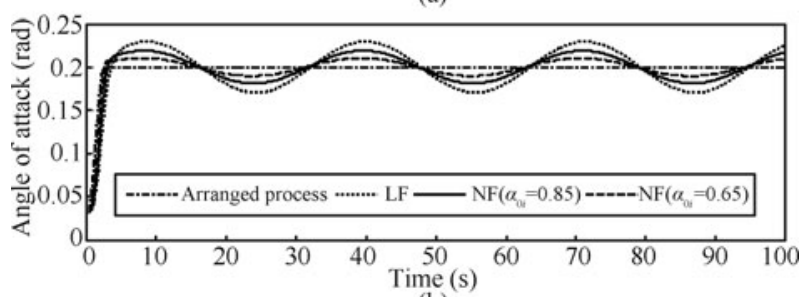

(b)

Fig. 8 Comparative simulation between NF and LF. (a) without disturbance; (b) with disturbance

\subsection{Monte Carlo simulation}

As for the other type of disturbances, i.e., the parametric uncertainty, a Monte Carlo simulation is executed for the integrated controller. Suppose ten parameters contain uncertainties, involving four inertia factors and six aerodynamic coefficients, which are the most important uncertain factors for clearance of flight control laws. These parameters are listed in Table 2.

In Monte Carlo analysis, the attitude commands are the same as (34). We respectively add $20 \%, 30 \%$, and $40 \%$ of normal distributed random uncertainties to the parameters listed in Table 2. Each group contains 500 runs. After each simulation, we verify the stability, dynamic process, and static performance to confirm whether the system is "well controlled". A probabilistic analysis is done to thoroughly evaluate the performance and robustness of the controller so as to offer a guideline to improve the control law. Table 3 
lists the probabilities of "well-controlled systems" with different uncertainty levels. The integrated controller shows good robustness to parametric uncertainty cases.

Table 2 Parameters with uncertainties

\begin{tabular}{cc}
\hline Uncertainty category & Parameter \\
\hline Inertia factor & Mass $(m)$ \\
Aerodynamic & Moments of inertia $\left(I_{x}, I_{y}, I_{z}\right)$ \\
Coefficient & Moment coefficients $\left(C_{L}, C_{D}, C_{Y}\right)$ \\
\hline
\end{tabular}

Table 3 The probabilities of well-controlled systems

\begin{tabular}{cc}
\hline $\begin{array}{c}\text { Uncertainty } \\
\text { level }\end{array}$ & $\begin{array}{c}\text { Probability of } \\
\text { well-controlled systems }\end{array}$ \\
\hline $20 \%$ & $499 / 500=99.8 \%$ \\
$30 \%$ & $488 / 500=97.6 \%$ \\
$40 \%$ & $479 / 500=95.8 \%$ \\
\hline
\end{tabular}

\subsection{Reference mission simulation}

In the reference mission, the guidance loop is included and the mission is to track the nominal trajectory defined in the velocity-altitude space, as described in Section 2.2. To make the test more demanding, again suppose that $\Delta_{\alpha}=0.03 \sin (0.2 t)$ and $\Delta_{\mu}=0.05 \sin (0.2 t)$ exist in the angle of attack and bank angle channels, respectively. In this aggressive case, the basic controller without ESO completely fails to stabilize the system, while the integrated controller still works well; see Fig. 9. The altitude tracking error is less than $100 \mathrm{~m}$ at the beginning and converges to almost zero in the end. The attitude angles chatter because of the disturbances, but they all stay within admissible ranges. We can also change initial entry states such as the initial vehicle position or attitude, and the controller shows good robustness under large initial entry condition dispersions.

\section{Conclusions}

In this work, an attitude controller that integrates dynamic inversion with active disturbance rejection control is designed for the GHV entry flight. Three ADRC components are adopted to improve the performance and robustness of the basic DI controller: ESO, ATP, and NF. By defining pseudo controls, the attitude system is divided into six first-order systems and each incorporates one ESO to estimate and compensate the disturbances: parametric uncertainties and modeling errors. Meanwhile, ATP is applied to generate reasonable reference commands and NF is adopted to improve the control performance and system robustness. The stability and estimation error of the nonlinear secondorder ESO are further discussed from a new but easier way where abundant qualitative properties of Liénard systems are utilized. It results in a comprehensive theoretic frame for nonlinear ESO stability and estimation error analysis, and offers a guideline to select the ESO parameters. Simulations have demonstrated the superiority of the integrated controller over the basic DI controller.
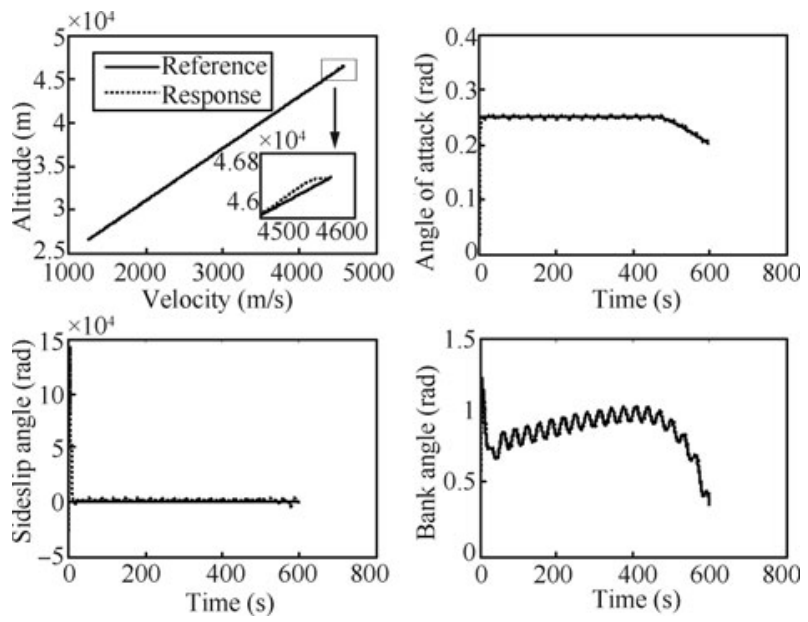

Fig. 9 Reference mission simulation results

\section{Acknowledgments}

The authors are grateful to the anonymous reviewers as well as to the Editor-in-Chief and Associate Editors for their insightful suggestions and kind encouragement.

\section{References}

[1] S. Bharadwaj, A. V. Rao, K. D. Mease. Entry trajectory tracking law via feedback linearization. Journal of Guidance, Control, and Dynamics, vol.21, no. 5, pp.726-732, 1998.

[2] E. Mooij, I. Barkana. Stability analysis of an adaptive guidance and control system applied to a winged re-entry vehicle. In Proceedings of AIAA Guidance, Navigation, and Control Conference, AIAA, San Francisco, CA, USA, pp. 4547-4559, 2005.

[3] L. Fiorentini, A. Serrani. Adaptive restricted trajectory tracking for a non-minimum phase hypersonic vehicle model. Automatica, vol. 48, no. 7, pp. 1248-1261, 2012.

[4] K. K. Gupta, L. S. Voelker. Aeroelastic simulation of hypersonic flight vehicles. AIAA Journal, vol. 50, no. 3, pp. 717$723,2012$.

[5] W. R. van Soest, Q. P. Chu, J. A. Mulder. Combined feedback linearization and constrained model predictive control for entry flight. Journal of Guidance, Control, and Dynamics, vol. 29, no. 2, pp. 427-434, 2006.

[6] Z. J. Shen, P. Lu. On-board entry trajectory planning expanded to sub-orbital flight. In Proceedings of AIAA Guidance, Navigation, and Control Conference and Exhibit, AIAA, Austin, Texas, USA, AIAA 2003-5736, 2003.

[7] W. J. Rugh, J. S. Shamma. Research on gain scheduling. Automatica, vol. 36, no. 10, pp. 1401-1425, 2000.

[8] D. Enns, D. Bugajski, R. Hendrick, G. Stein. Dynamic inversion: An evolving methodology for flight control design. International Journal of Control, vol. 59, no. 1, pp. 71-91, 1994.

[9] D. Ito, J. Georgie, J. Valasek, D. T. Ward. Reentry Vehicle Flight Controls Design Guidelines: Dynamic Inversion, 
Final Technical Report NASA TP-2002-210771, Flight Simulation Laboratory, Texas Engineering Experiment Station Texas A\&M University, USA, 2002.

[10] H. J. Xu, M. D. Mirmirani, P. A. Ioannou. Adaptive sliding mode control design for a hypersonic flight vehicle. Journal of Guidance, Control, and Dynamics, vol. 27, no. 5, pp. 829838, 2004.

[11] A. Rahideh, A. H. Bajodah, M. H. Shaheed. Real time adaptive nonlinear model inversion control of a twin rotor MIMO system using neural networks. Engineering Applications of Artificial Intelligence, vol. 25, no. 6, pp. 1289-1297, 2012.

[12] Z. Q. Gao, Y. Huang, J. Q. Han. An alternative paradigm for control system design. In Proceedings of the 40th IEEE Conference on Design and Control, IEEE, Orlando, Florida, USA, pp. 4578-4585, 2001.

[13] J. Q. Han. From PID to active disturbance rejection control. IEEE Transactions on Industrial Electronics, vol. 56, no. 3, pp. 900-906, 2009.

[14] J. Q. Han. Active Disturbance Rejection Control Technique - The Technique for Estimating and Compensating the Uncertainties, Beijing: National Defense Industry Press, 2008. (in Chinese)

[15] B. Z. Guo, Z. L. Zhao. On convergence of the nonlinear active disturbance rejection control for MIMO systems. SIAM Journal on Control and Optimization, vol. 51, no. 2, pp. 1727-1757, 2013.

[16] Q. Zheng, Z. Z. Chen, Z. Q. Gao. A practical approach to disturbance decoupling control. Control Engineering Practice, vol. 17, no. 9, pp. 1016-1025, 2009.

[17] R. Kotina, Q. Zheng, A. J. van den Bogert, Z. Q. Gao. Active disturbance rejection control for human postural sway. In Proceedings of American Control Conference, IEEE, San Francisco, CA, USA, pp. 4081-4086, 2011.

[18] F. Léonard, A. Martini, G. Abba. Robust nonlinear controls of model-scale helicopters under lateral and vertical wind gusts. IEEE Transactions on Control Systems Technology, vol. 20, no. 1, pp. 154-163, 2012.

[19] Z. Ren, J. F. Fan, J. J. Li. A new robust controller for flight control system of hypersonic flying vehicle. Advanced Materials Research, vol. 562-564, pp. 1682-1688, 2012.

[20] Y. G. Zhu, G. L. Fan, J. Q. Yi. Controller design for flying boats taking off from water with regular waves. In Proceedings of 2012 IEEE International Conference on Mechatronics and Automation, Chengdu, China, pp. 480-485, 2012.

[21] X. X. Fang, Q. Lin, Y. X. Wang, L. L. Zheng. Control strategy design for the transitional mode of tiltrotor UAV. In Proceedings of the 10th IEEE International Conference on Industrial Informatics, Beijing, China, pp. 248-253, 2012.

[22] J. D. Shaughnessy, S. Z. Pinckney, J. D. Mcminn. Hypersonic Vehicle Simulation Model: Winged-cone Configuration, Technical Report NASA TM-102610, NASA Langley Research Center, USA, 1990.
[23] S. Keshmiri, R. Colgren, M. Mirmirani. Development of an aerodynamic database for a generic hypersonic air vehicle. In Proceedings of AIAA Guidance, Navigation, and Control Conference and Exhibit, AIAA, San Francisco, CA, USA, AIAA 2005-6257, 2005.

[24] S. Keshmiri, R. Colgren, M. Mirmirani. Six-DOF modeling and simulation of a generic hypersonic vehicle for control and navigation purposes. In Proceedings of AIAA Guidance, Navigation, and Control Conference and Exhibit, AIAA, Keystone, Colorado, USA, pp. 4680-4689, 2006.

[25] J. Mawhin, J. R. Ward Jr. Periodic solutions of some forced Liénard differential equations at resonance. Archiv der Mathematik, vol. 41, no. 4, pp.337-351, 1983.

[26] J. Sugie, Y. Amano. Global asymptotic stability of nonautonomous systems of Liénard type. Journal of Mathematical Analysis and Applications, vol.289, no. 2, pp.673-690, 2004 .

[27] C. Tunç, E. Tunç. On the asymptotic behavior of solutions of certain second-order differential equations. Journal of the Franklin Institute, vol. 344, no. 5, pp. 391-398, 2007.

[28] Y. Huang, J. Q. Han. The self-stable region approach for second order nonlinear uncertain systems. In Proceedings of 1999 IFAC World Congress, IFAC, Beijing, China, pp. 135140, 1999.

[29] J. Q. Han, R. Zhang. Error analysis of the second order ESO. Journal of Systems Science and Mathematical Sciences, vol. 19, no. 4, pp. 465-471, 1999.

[30] Z. X. Gan, J. Q. Han. Construction of Lyapunov function for 2-order ESO. In Proceedings of the 21st Chinese Control Conference, Beijing, China, pp. 354-357, 2002. (in Chinese)

[31] S. A. Snell, D. F. Enns, W. L. Garrard Jr. Nonlinear inversion flight control for a supermaneuverable aircraft. Journal of Guidance, Control, and Dynamics, vol. 15, no. 4, pp. 976984, 1992.

[32] Z. Q. Pu, X. M. Tan, G. L. Fan, J. Q. Yi. Design of entry trajectory tracking law for suborbital hypersonic vehicle via inversion control. In Proceedings of the 10th World Congress on Intelligent Control and Automation, Beijing, China, pp. 1092-1097, 2012.

[33] H. K. Khalil. Nonlinear Systems, 3rd ed., Upper Saddle River, New Jersey: Prentice Hall Press, 2001.

[34] Q. Zheng, L. Q. Gao, Z. Q. Gao. On stability analysis of active disturbance rejection control for nonlinear time-varying plants with unknown dynamics. In Proceedings of the 46th IEEE Conference on Decision and Control, IEEE, New Orleans, LA, USA, pp. 3501-3506, 2007.

[35] W. K. Zhou, S. Shao, Z. Q. Gao. A stability study of the active disturbance rejection control problem by a singular perturbation approach. Applied Mathematical Sciences, vol. 3, no. 10, pp. 491-508, 2009.

[36] T. Yoshizawa. Stability Theory and the Existence of Periodic Solutions and Almost Periodic Solutions, New York: Springer-Verlag, 1975. 
[37] G. E. H. Reuter. A boundedness theorem for nonlinear differential equations of the second order. Mathematical Proceedings of the Cambridge Philosophical Society, vol.47, no. 1, pp. 49-54, 1951.

[38] A. Kroopnick. Properties of solutions to a generalized Liénard equation with forcing term. Applied Mathematics E-Notes, vol. 8, pp. 40-44, 2008.

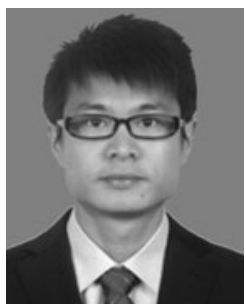

Zhi-Qiang Pu received his B. Eng. degree from Wuhan University in 2009. He is currently a Ph. D. candidate in Institute of Automation, Chinese Academy of Sciences, China.

His research interests include nonlinear robust control and adaptive control, especially the guidance and control of hypersonic vehicles.

E-mail: zhiqiang.pu@ia.ac.cn

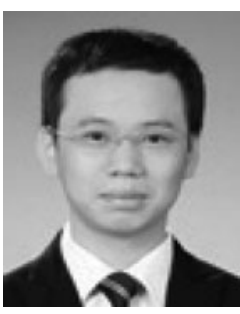

Ru-Yi Yuan received his B. Eng. degree from Hunan University in 2006 and $\mathrm{Ph} . \mathrm{D}$. degree from Institute of Automation, Chinese Academy of Sciences, China in 2011. He is currently a research assistant in the Integrated Information System Research Center, Institute of Automation.

His research interests include nonlinear control, artificial intelligence, and flight control.

E-mail: ruyi.yuan@ia.ac.cn (Corresponding author)

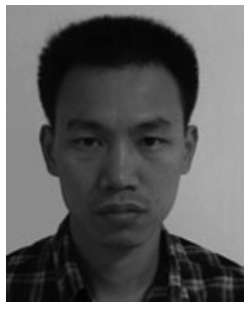

Xiang-Min Tan received his B.Eng. degree from Central South University in 2004 and Ph. D. degree from Institute of Automation, Chinese Academy of Sciences, China in 2009. He is currently a senior engineer in the Integrated Information System Research Center, Institute of Automation.

His research interests include hypersonic dustrial control, and neural networks.

E-mail: xiangmin.tan@ia.ac.cn

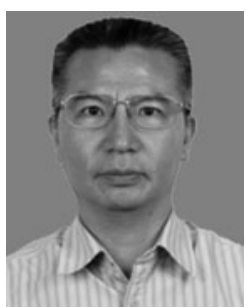

Jian-Qiang Yi received his B. Eng. degree from the Beijing Institute of Technology, Beijing, China in 1985, and the M. Eng. and Ph. D. degrees from the Kyushu Institute of Technology, Kitakyushu, Japan in 1989 and 1992, respectively. He worked as a research fellow at the Computer Software Development Company, Tokyo, Japan from 1992 to 1994, and a chief engineer at MYCOM, Inc., Kyoto, Japan from 1994 to 2001. Since 2001 he has been with the Institute of Automation, Chinese Academy of Sciences, China, where he is currently a professor. He is an associate editor for the IEEE Computational Intelligence Magazine, Journal of Advanced Computational Intelligence and Intelligent Informatics, and Journal of Innovative Computing, Information and Control.

His research interests include theories and applications of intelligent control, intelligent robotics, underactuated system control, sliding-mode control, and flight control.

E-mail: jianqiang.yi@ia.ac.cn 